\title{
Modification of the effect of smoking on cholesterol in Japanese carriers of a PTPN11 polymorphism
}

\author{
RIEKO OKADA $^{1}$, KOJI SUZUKI ${ }^{2}$, KAZUKO NISHIO $^{1}$, YOSHIKO ISHIDA $^{1}$, SAYO KAWAI $^{1}$, YASUYUKI GOTO $^{1}$, \\ MARIKO NAITO $^{1}$, KENJI WAKAI ${ }^{1}$, YOSHINORI ITO ${ }^{1}$ and NOBUYUKI HAMAJIMA ${ }^{1}$ \\ ${ }^{1}$ Department of Preventive Medicine/Biostatistics and Medical Decision Making, Nagoya University Graduate School of \\ Medicine, 65 Tsurumai-cho, Showa-ku, Nagoya; ${ }^{2}$ Department of Public Health, Fujita Health University \\ School of Health Sciences, 1-98 Dengakugakubo, Kutsukake-cho, Toyoake, Aichi, Japan
}

Received March 28, 2008; Accepted May 20, 2008

\begin{abstract}
A widely-expressed protein tyrosine phosphatase, SHP-2, regulates apoB secretion by insulin. We hypothesised that a variation in the SHP-2 gene, PTPN11, may interact with smoking to influence serum lipid concentrations. The study comprised 794 subjects (278 males and 516 females), aged 39-88 years, who attended a health examination in Hokkaido, Japan in 2003. Subjects were genotyped for a $G / A$ PTPN11 polymorphism (rs2301756). The mean serum LDL cholesterol, HDL cholesterol and triglyceride levels, stratified by genotype, were compared between current and non- (never or ex-) smokers. Among the $A A$ genotype carriers, LDL cholesterol levels were significantly decreased in current vs. nonsmokers $[$ mean $\pm \mathrm{SD}, 101 \pm 34 \mathrm{mg} / \mathrm{dl}$ vs. $146 \pm 40 \mathrm{mg} / \mathrm{dl} ; b$ (adjusted mean difference) $=-56.0 ; 95 \%$ CI -102.8 to -9.3 ; $\mathrm{p}=0.019$ after adjustment for age, sex and body mass index]. In contrast, HDL cholesterol levels were increased in current vs. non-smokers $(66 \pm 5 \mathrm{mg} / \mathrm{dl}$ vs. $58 \pm 12 \mathrm{mg} / \mathrm{dl} ; b=14.9 ; 95 \% \mathrm{CI}$ 3.1 to $26.8 ; \mathrm{p}=0.014$ after adjustment). The interaction between a current smoking habit and the $A A$ genotype had a significant effect on LDL and HDL cholesterol (LDL-C, $\mathrm{p}=0.039$; HDL-C, $\mathrm{p}=0.020$ ). These data suggest that cigarette smoking might alter the metabolism of cholesterol in Japanese PTPN11 $A A$ genotype carriers.
\end{abstract}

\section{Introduction}

Cigarette smoking and an increased level of LDL cholesterol (LDL-C) or decreased level of HDL cholesterol (HDL-C) are risk factors for cardiovascular disease. Increases in LDL-C

Correspondence to: Dr Rieko Okada, Department of Preventive Medicine/Biostatistics and Medical Decision Making, Nagoya University Graduate School of Medicine, 65 Tsurumai-cho, Showaku, Nagoya 466-8550, Japan

E-mail: rieokada@med.nagoya-u.ac.jp

Key words: Src homology 2 domain-containing protein tyrosine phosphatase-2, LDL cholesterol, HDL cholesterol, smoking, PTPN11, genetic polymorphism levels and decreases in HDL-C levels have been observed in smokers (1), and a positive multiplicative interaction between smoking and LDL-C levels in coronary heart disease incidence has been observed (2). Cigarette smoking is an oxidative stress that causes genetic damage (3) and can either up- or downregulate gene expression levels (4). Studies have tried to clarify the effect of the gene-smoking interaction on lipid concentrations $(5,6)$, and have revealed that both genetic variants and environmental factors, including a current smoking habit, influence serum cholesterol levels (7).

Src homology 2 domain-containing protein tyrosine phosphatase-2 (SHP-2) is a widely-expressed protein tyrosine phosphatase (8) that regulates both acute and adaptive control of apoB secretion by insulin (9-11). The PTPN11 gene, which encodes SHP-2, is on chromosome 12 and contains 16 exons. Jamshidi et al selected three tagging single nucleotide polymorphisms (SNPs), rs11066322, rs11066320 and rs2301756, and demonstrated in a Caucasian female population that PTPN11 rs11066322 was associated with apoB levels and rs11066320 with LDL-C levels (12). However, the NCBI Variation Database shows that, in the Japanese population, SNP rs 11066320 does not exist or is very rare, while rs 11066322 and rs2301756 are in nearly complete linkage disequilibrium with each other. We therefore selected rs2301756, (IMS-JST057927 in the Japan Single Nucleotide Polymorphism database), located in intron 3, 223-bp upstream of exon 4, as a candidate SNP influencing LDL-C levels among the Japanese.

\section{Patients and methods}

Study population. Annual health examinations have been available to inhabitants in a rural area of Hokkaido, Japan since 1982. A total of 864 examinees (309 males and 555 females) aged 39-88 years who attended a health examination in August 2003 were considered for the present polymorphism study. Of these, 60 subjects who did not agree to participate, 7 subjects whose genotype analyses were not successful and 3 subjects whose smoking status could not be determined were excluded. As well, 91 subjects taking lipid-lowering medication were not included in the analysis of lipid profiles. The remaining 703 subjects (257 males and 446 females), aged 39-88 years, were included in the study. Written informed consent was obtained from each participant, and the study was approved by the 
Table I. Characteristics of the 794 subjects according to PTPN11 genotype.

\begin{tabular}{|c|c|c|c|c|}
\hline & $\begin{array}{c}G G \\
\mathrm{n}=493\end{array}$ & $\begin{array}{c}G A \\
\mathrm{n}=261\end{array}$ & $\begin{array}{c}A A \\
\mathrm{n}=40\end{array}$ & p-value \\
\hline Age (year, mean $\pm \mathrm{SD})$ & $62 \pm 10$ & $60 \pm 10$ & $63 \pm 9$ & 0.151 \\
\hline Male (\%) & $167(34)$ & $100(38)$ & $11(28)$ & 0.283 \\
\hline Body mass index $\left(\mathrm{kg} / \mathrm{m}^{2}\right.$, mean $\left.\pm \mathrm{SD}\right)$ & $24 \pm 3$ & $24 \pm 3$ & $24 \pm 3$ & 0.218 \\
\hline Use of lipid lowering medications (\%) & $54(11)$ & $33(13)$ & $4(10)$ & 0.752 \\
\hline Ex-smokers $(\%)$ & $115(23)$ & $63(24)$ & $6(15)$ & 0.440 \\
\hline Current smokers (\%) & $76(15)$ & $51(20)$ & $4(10)$ & 0.183 \\
\hline
\end{tabular}

${ }^{\mathrm{a} C a l c u l a t e d}$ by analysis of variance or a 2 by $3 \chi^{2}$ test.

Ethics Committee of Nagoya University School of Medicine (approval no. 48).

Biochemical analysis and smoking status. Blood sample collection for determining fasting lipids was carried out on subjects after a minimum 8-h overnight fast. A colourimetric enzymatic method was used to determine total cholesterol, HDL-C and triglyceride levels using an autoanalyzer (L-type Wako, CHO.M, HDL-C.M, TG.M, Tokyo, Japan). The Friedewald equation (13) was used to calculate LDL-C levels in subjects with triglycerides $<400 \mathrm{mg} / \mathrm{dl}(\mathrm{n}=696)$ :

\section{$[\mathrm{LDL}-\mathrm{C}(\mathrm{mg} / \mathrm{dl})]=[$ total cholesterol $(\mathrm{mg} / \mathrm{dl})]-$ [HDL-C (mg/dl)] - [triglyceride $(\mathrm{mg} / \mathrm{dl})] / 5$}

Smoking status was determined by a standardised questionnaire and defined as current or non- (never or ex-) smoker.

Genotyping. DNA was extracted from whole blood using a BioRobot EZ1 (Qiagen Group, Tokyo, Japan). An SNP in intron 3 of PTPN11 (rs2301756) was genotyped by polymerase chain reaction with confronting two-pair primers (PCR-CTPP) (14). The primers were: F1 (5'-GATTGGGCA ATGGACGA) and R1 (5'-AATGACCACTAAACTTCTTAA ATGAGC); F2 (5'-CATTTGTCTCTAAAGGACTGTGGA) and R2 (5'-AATCTGCATCCCATGCAG) (15). Genomic DNA was added to a volume of $25 \mu 1$ with $0.12 \mathrm{mM}$ dNTPs, 25 pmol of each primer, 0.5 units of AmpliTaq Gold (PerkinElmer, Foster City, CA) and 2.5 $\mu 1$ 10X PCR buffer including $15 \mathrm{mM} \mathrm{MgCl}_{2}$. PCR was performed with initial denaturation at $95^{\circ} \mathrm{C}$ for $10 \mathrm{~min}$, followed by 30 cycles of denaturation at $95^{\circ} \mathrm{C}$ for $1 \mathrm{~min}$, annealing at $60^{\circ} \mathrm{C}$ for $1 \mathrm{~min}$ and extension at $72^{\circ} \mathrm{C}$ for $5 \mathrm{~min}$. The final extension was conducted at $72^{\circ} \mathrm{C}$ for $5 \mathrm{~min}$. The PCR product was visualised on a $2 \%$ agarose gel with ethidium bromide staining. $G$ and $A$ alleles were identified by the presence of $125 \mathrm{bp}$ and $94 \mathrm{bp}$ bands, respectively.

Statistical analysis. Background characteristics were compared among subjects with the $G G, G A$ and $A A$ genotypes using the analysis of variance or the $\chi^{2}$ test. Triglyceride levels were transformed to their logarithms to improve the normality of distribution, and the geometric means and inter-quartile range were given. The mean serum LDL-C and HDL-C and the geometric mean of triglyceride levels were stratified by genotype. These serum lipid levels, adjusted for age, sex and body mass index (BMI) by multiple linear regression models, were then compared in current and non-smokers. The bootstrap method with 1000 replications was used to derive a $95 \%$ confidence interval (CI) using the bias corrected and accelerated (BCa) method, which was shown to be appropriate for small sample sizes because of its high internal and external validity (16). The effect of interactions between a current smoking habit and the $A A$ genotype on LDL-C and HDL-C levels, adjusted for age, sex and BMI, was calculated, and the genotype distribution of the PTPN11 polymorphism was tested for Hardy-Weinberg equilibrium. STATA Version 9 (STATA Corp., College Station, TX) was used for these analyses.

\section{Results}

The characteristics of the subjects according to PTPN11 genotype are shown in Table I. Of the subjects, 62, 33 and 5\% had the $G G, G A$ and $A A$ genotype, respectively. This distribution was in Hardy-Weinberg equilibrium $\left(\chi^{2}=0.41, \mathrm{p}=0.521\right)$. There were no significant differences among the three genotypes in terms of age, sex, BMI, proportion of subjects with lipidlowering medication, or ex- or current smokers.

The mean serum levels of LDL-C and HDL-C and the geometric mean of triglyceride levels according to the PTPN11 polymorphism and smoking status are shown in Table II. Among the $A A$ genotype carriers, a decreased level of LDL-C was observed in current smokers vs. non-smokers (mean $\pm \mathrm{SD}$, $101 \pm 34 \mathrm{mg} / \mathrm{dl}$ vs. $146 \pm 40 \mathrm{mg} / \mathrm{dl}$ ). This difference was statistically significant after adjustment for age, sex and BMI $[b$ (adjusted mean difference $)=-56.0 ; 95 \%$ CI -102.8 to -9.3 ; $\mathrm{p}=0.019$ ) (Table III). Among GA genotype carriers, decreased levels of LDL-C were also observed in current vs. non-smokers $(130 \pm 33 \mathrm{mg} / \mathrm{dl}$ vs. $139 \pm 29 \mathrm{mg} / \mathrm{dl})$, but this difference did not retain statistical significance after adjustment. Such a difference was not observed among $G G$ genotype carriers. As well, the mean HDL-C level was increased in current smokers with the $A A$ genotype, which was not observed among the other genotype carriers. The effect of interactions between a current smoking habit and the $A A$ genotype on LDL-C and HDL-C were significant (LDL-C: interaction $-22.8,95 \% \mathrm{CI}-42.5$ to -3.2, $\mathrm{p}=0.023$; HDL-C: interaction $16.4,95 \%$ CI 2.6 to 30.2 , $\mathrm{p}=0.020)$. The difference in the geometric mean of triglyceride levels between current and non-smokers was not significant for any genotype (data not shown). 
Table II. Mean LDL cholesterol ( $\mathrm{n}=696)$, HDL cholesterol $(\mathrm{n}=703)$ and triglyceride levels $(\mathrm{n}=703)$ according to PTPN11 genotype and smoking status.

\begin{tabular}{|c|c|c|c|c|}
\hline Genotype & Total & Never smokers & Ex-smokers & Current smokers \\
\hline \multicolumn{5}{|c|}{ LDL cholesterol $(\mathrm{mg} / \mathrm{dl})$, mean $\pm \mathrm{SD}(\mathrm{n})$} \\
\hline$G G$ & $135 \pm 30(434)$ & $138 \pm 29(263)$ & $133 \pm 32(102)$ & $129 \pm 28(69)$ \\
\hline$G A$ & $137 \pm 30(226)$ & $141 \pm 29(121)$ & $137 \pm 28 \quad(55)$ & $130 \pm 33(50)$ \\
\hline$A A$ & $141 \pm 41 \quad(36)$ & $145 \pm 41 \quad(27)$ & $151 \pm 35 \quad(5)$ & $101 \pm 34$ \\
\hline \multicolumn{5}{|c|}{ HDL cholesterol (mg/dl), mean \pm SD (n) } \\
\hline$G G$ & $59 \pm 13(439)$ & $60 \pm 13(264)$ & $56 \pm 13(105)$ & $55 \pm 12(70)$ \\
\hline$G A$ & $59 \pm 15(228)$ & $61 \pm 15(121)$ & $58 \pm 12 \quad(57)$ & $53 \pm 15(50)$ \\
\hline$A A$ & $59 \pm 12$ & $60 \pm 11$ & $47 \pm 14$ & $66 \pm 5$ \\
\hline \multicolumn{5}{|c|}{ Triglyceride (mg/dl), geometric mean (inter-quartile range) (n) } \\
\hline$G G$ & $88(65-116)(439)$ & $83(62-112)(264)$ & $98(68-126)(105)$ & $95(67-123)(70)$ \\
\hline$G A$ & $90(66-115)(228)$ & $85(66-104)(121)$ & $94(61-131) \quad(57)$ & $96(67-135)(50)$ \\
\hline$A A$ & $89(65-114) \quad(36)$ & $91(62-117) \quad(27)$ & $77(72-95)$ & $95(84-108) \quad(4)$ \\
\hline
\end{tabular}

Subjects taking lipid-lowering medication were excluded. SD, standard deviation.

Table III. Mean LDL ( $\mathrm{n}=696)$ and HDL ( $\mathrm{n}=703)$ cholesterol levels in current vs. non-smokers according to PTPN11 genotype.

\begin{tabular}{|c|c|c|c|c|c|}
\hline Genotype & $\begin{array}{c}\text { Non-smokers } \\
\text { mean } \pm \text { SD (No.) }\end{array}$ & $\begin{array}{l}\text { Current smokers } \\
\text { mean } \pm \text { SD (No.) }\end{array}$ & $b^{\mathrm{a}}$ & $\left(95 \% \mathrm{CI}^{\mathrm{a}}\right)$ & $\mathrm{p}$-value ${ }^{\mathrm{a}}$ \\
\hline \multicolumn{6}{|c|}{ LDL cholesterol (mg/dl) } \\
\hline$G G$ & $137 \pm 30(365)$ & $129 \pm 28(69)$ & -4.1 & $(-11.3$ to 3.1$)$ & 0.260 \\
\hline$G A$ & $139 \pm 29(176)$ & $130 \pm 33(50)$ & -9.0 & $(-19.2$ to 1.3$)$ & 0.086 \\
\hline$A A$ & $146 \pm 40 \quad(32)$ & $101 \pm 34$ & -56.0 & $(-102.8$ to -9.3$)$ & 0.019 \\
\hline$A A+G A$ & $139 \pm 29(208)$ & $130 \pm 33(54)$ & -11.8 & $(-21.7$ to -1.9$)$ & 0.019 \\
\hline \multicolumn{6}{|c|}{ HDL cholesterol (mg/dl) } \\
\hline$G G$ & $59 \pm 14(369)$ & $55 \pm 12(70)$ & -3.7 & $(-6.9$ to -0.5$)$ & 0.024 \\
\hline$G A$ & $60 \pm 14(178)$ & $53 \pm 15(50)$ & -6.2 & $(-11.2$ to -1.2$)$ & 0.016 \\
\hline$A A$ & $58 \pm 12$ & $66 \pm 5$ & 14.9 & (3.1 to 26.8$)$ & 0.014 \\
\hline$A A+G A$ & $60 \pm 14(210)$ & $53 \pm 15(54)$ & -5.1 & $(-10.0$ to -0.2$)$ & 0.043 \\
\hline
\end{tabular}

${ }^{a}$ Adjusted for age, sex and BMI by multivariate regression models with bootstrap method. Non-smoker, never or ex-smoker; $b$, coefficient; CI, confidence interval.

\section{Discussion}

The results of this study demonstrate that the rs2301756 polymorphism of the PTPN11 gene (encoding SHP-2) interacts with smoking to modify LDL-C and HDL-C levels. In the case of the PTPN11 AA genotype, a significant decrease in LDL-C levels and increase in HDL-C levels were observed in current smokers compared to non-smokers.

A recent study by Jamshidi et al (12) revealed that PTPN11 rs 11066320 was associated with LDL-C levels in a Caucasian female population. However, this SNP does not exist or is very rare in the Japanese population. It is therefore possible that rs2301756, another SNP that is prevalent among the Japanese, might determine LDL-C levels in a Japanese population, if functional. Another study showed the cholesterol-dependent binding of SHP-2 to membranes, which represents a mechanism for maintaining junctional stability (17). Consequently, based on experimental and epidemiological studies demonstrating the association between SHP-2 and cholesterol levels, our hypothesis that the SHP-2 gene polymorphism might be related to cholesterol levels warranted investigation.

Previous studies have shown an increase in LDL-C and a decrease in HDL-C levels among smokers in Caucasian populations (1). Though an increase in LDL-C levels has not been observed in a Japanese population (18), a positive multiplicative interaction between smoking and LDL-C in coronary heart disease incidence has been reported (2). The relative risk for coronary heart disease in current smokers and non- 
smokers was 2.91 in subjects with an LDL-C of 130-159 mg/dl, and only 1.33 in subjects with an LDL-C below $100 \mathrm{mg} / \mathrm{dl}$. The study showed that the mean LDL-C level was only $101 \mathrm{mg} / \mathrm{dl}$ in subjects with the $A A$ genotype who smoked. Whether or not cardiovascular risk is less for $A A$ genotype smokers compared to smokers of other genotypes must be validated by prospective studies, and the cessation of smoking remains of definite importance for carriers of any genotype.

It is known that the deregulation of SHP-2 by Helicobacter pylori CagA protein may induce abnormal proliferation of gastric epithelial cells and cause gastric cancer (19). We have shown that both Japanese (15) and Japanese-Brazilian (20) subjects with the $A A$ genotype have a reduced risk of gastric atrophy as compared to $G G$ genotype carriers. This suggests that the $A A$ genotype might be related to the formation of a less active form of SHP-2. Molecular analysis must be conducted to determine whether this polymorphism is itself functional, or whether it is linked to another functional polymorphism, thus influencing SHP-2 activity.

The percentages of subjects in this study with the $G G, G A$ and $A A$ genotype were 62,33 and $5 \%$, respectively. These are similar to the percentages found in our two previous studies of Japanese (68, 29 and 4\%) (15) and Japanese-Brazilian (66, 30 and 4\%) (20) populations. Data from the HapMap database show that the $G$ allele is dominant in Asians, while the $A$ allele is dominant in Caucasians and African Americans. As allele frequency is completely different among populations, and as another SNP (rs11066320) might partly determine serum LDL-C levels in Caucasians (12), this interaction of smoking and the $A A$ genotype, with effects on cholesterol levels, may not be observed in Caucasians.

In summary, the polymorphism rs2301756 in the PTPN11 gene encoding SHP-2 interacts with smoking, thus influencing LDL-C and HDL-C levels. Among PTPN11 AA genotype carriers, a significant decrease in LDL-C and increase in HDL-C levels were observed in current smokers. These data suggest that cigarette smoking might alter the metabolism of cholesterol in PTPN11 AA genotype carriers.

\section{Acknowledgements}

The authors are grateful to Emeritus Professor Kunio Aoki, who established the present field of research in 1982 and chaired the research group until 2003, to Ms. Yoko Mitsuda for her technical assistance, and to the staff members of the health examination program of Y-town, Hokkaido, Japan. This study was supported in part by a Grant-in-Aid for Scientific Research on Special Priority Areas of Cancer from the Japanese Ministry of Education, Culture, Sports, Science and Technology.

\section{References}

1. Craig WY, Palomaki GE and Haddow JE: Cigarette smoking and serum lipid and lipoprotein concentrations: an analysis of published data. BMJ 298: 784-788, 1989.
2. Hozawa A, Folsom AR, Sharrett AR, Payne TJ and Chambless LE: Does the impact of smoking on coronary heart disease differ by low-density lipoprotein cholesterol level?: the Atherosclerosis Risk in Communities (ARIC) Study. Circ J 70: 1105-1110, 2006.

3. Gabriel HE, Crott JW, Ghandour H, et al: Chronic cigarette smoking is associated with diminished folate status, altered folate form distribution, and increased genetic damage in the buccal mucosa of healthy adults. Am J Clin Nutr 83: 835-841, 2006.

4. Philibert RA, Sandhu H, Hollenbeck N, Gunter T, Adams W and Madan A: Transcriptional profiling of subjects from the Iowa adoption studies. Am J Med Genet B Neuropsychiatr Genet 144: 683-690, 2007.

5. Costanza MC, Cayanis E, Ross BM, Flaherty MS, Alvin GB, Das K and Morabia A: Relative contributions of genes, environment, and interactions to blood lipid concentrations in a general adult population. Am J Epidemiol 161: 714-724, 2005.

6. Czerwinski SA, Mahaney MC, Rainwater DL, Vandeberg JL, MacCluer JW, Stern MP and Blangero J: Gene by smoking interaction: evidence for effects on low-density lipoprotein size and plasma levels of triglyceride and high-density lipoprotein cholesterol. Hum Biol 76: 863-876, 2004.

7. Morabia A, Cayanis E, Costanza MC, et al: Association of extreme blood lipid profile phenotypic variation with 11 reverse cholesterol transport genes and 10 non-genetic cardiovascular disease risk factors. Hum Mol Genet 12: 2733-2743, 2003.

8. Tiganis T and Bennett AM: Protein tyrosine phosphatase function: the substrate perspective. Biochem J 402: 1-15, 2007

9. Phung TL, Roncone A, Jensen KL, Sparks CE and Sparks JD: Phosphoinositide 3-kinase activity is necessary for insulindependent inhibition of apolipoprotein B secretion by rat hepatocytes and localizes to the endoplasmic reticulum. J Biol Chem 272: 30693-30702, 1997.

10. Au WS, Kung HF and Lin MC: Regulation of microsomal triglyceride transfer protein gene by insulin in HepG2 cells: roles of MAPKerk and MAPKp38. Diabetes 52: 1073-1080, 2003.

11. Ugi S, Maegawa H, Kashiwagi A, Adachi M, Olefsky JM and Kikkawa R: Expression of dominant negative mutant SHPTP2 attenuates phosphatidylinositol 3'-kinase activity via modulation of phosphorylation of insulin receptor substrate-1. J Biol Chem 271: 12595-12602, 1996.

12. Jamshidi Y, Gooljar SB, Snieder H, et al: SHP-2 and PI3-kinase genes PTPN11 and PIK3R1 may influence serum apoB and LDL cholesterol levels in normal women. Atherosclerosis 194: e26-e33, 2007.

13. Friedewald WT, Levy RI and Fredrickson DS: Estimation of the concentration of low-density lipoprotein cholesterol in plasma, without use of the preparative ultracentrifuge. Clin Chem 18: 499-502, 1972.

14. Hamajima N, Saito T, Matsuo K, Kozaki K, Takahashi T and Tajima K: Polymerase chain reaction with confronting two-pair primers for polymorphism genotyping. Jpn J Cancer Res 91: 865-868, 2000.

15. Goto Y, Ando T, Yamamoto K, Tamakoshi A, El-Omar E, Goto H and Hamajima N: Association between serum pepsinogens and polymorphism of PTPN11 encoding SHP-2 among Helicobacter pylori seropositive Japanese. Int J Cancer 118: 203-208, 2006.

16. Steyerberg EW, Bleeker SE, Moll HA, Grobbee DE and Moons KG: Internal and external validation of predictive models: a simulation study of bias and precision in small samples. J Clin Epidemiol 56: 441-447, 2003.

17. Burkart A, Samii B, Corvera S and Shpetner HS: Regulation of the SHP-2 tyrosine phosphatase by a novel cholesterol- and cell confluence-dependent mechanism. J Biol Chem 278: 18360-18367, 2003.

18. Choudhury SR, Ueshima H, Kita Y, et al: Alcohol intake and serum lipids in a Japanese population. Int J Epidemiol 23: 940-947, 1994.

19. Higashi H, Tsutsumi R, Muto S, Sugiyama T, Azuma T, Asaka M and Hatakeyama M: SHP-2 tyrosine phosphatase as an intracellular target of Helicobacter pylori CagA protein. Science 295: 683-686, 2002.

20. Kawai S, Goto Y, Ito LS, et al: Significant association between PTPN11 polymorphism and gastric atrophy among Japanese Brazilians. Gastric Cancer 9: 277-283, 2006. 